\title{
Crédito de desarrollo humano: diagnóstico de los últimos nueve años y su incidencia en las familias de extrema pobreza de la zona 8 Guayaquil-Ecuador
}

\section{Human development credit: diagnosis of the last nine years and its impact on extreme poverty families in zone 8 Guayaquil-Ecuador}

\author{
Guillermo Castillo Tumaille \\ Patricia Salazar Torres \\ Jessica Ivonne Caguana Baquerizo \\ Universidad de Guayaquil, Ecuador \\ Autor para correspondencia: Guillermo.castillotu@ug.edu.ec, patricia.salazarto@ug.edu.ec, \\ Jessica.caguanab@ug.edu.ec \\ Fecha de recepción: 03 de Mayo de 2017 - Fecha de aceptación: 10 de Agosto de 2017
}

Resumen: En América Latina se han producido sustanciales cambios, como también en Ecuador en los últimos años se han experimentado variaciones significativas en el desarrollo económico y social. Este artículo tiene como objetivo verificar el comportamiento del Crédito de Desarrollo Humano $(\mathrm{CDH})$, entregados a las familias de extrema pobreza de la Zona 8 (Guayaquil Norte- Sur y Durán). La metodología que se utilizó fue la recopilación de información por parte del Ministerio de Inclusión Económica y Social (MIES) Zona 8, sobre los créditos realizados en los últimos nueve años, con un análisis de los datos para concluir con una explicación de la tendencia de los créditos de Desarrollo Humano, donde los resultados resaltan la zona, distrito, tipo, subsidio, sexo, edad y actividad de las familias que recibieron el Crédito de Desarrollo Humano. De los resultados tenemos que el 94\% son mujeres y el $6 \%$ son hombres, la edad del $75 \%$ de las madres están entre 18 y 40 años, la mayor actividad está en el $58 \%$ en venta de ropa, el $25 \%$ en cría de ave de corral, cría de ganado bovino, acuicultura marina, pesca marina y el $13 \%$ en venta de comida.

Palabras clave: crédito de desarrollo humano asociativo (CDHA); economía popular y solidaria; inclusión social; emprendimiento

\begin{abstract}
In Latin America there have been substantial changes, as in Ecuador in recent years there have been significant variations in economic and social development. This article aims to verify the behavior of the Human Development Credit $(\mathrm{CDH})$, delivered to the extreme poverty families of Zone 8 (Guayaquil North-South and Durán). The methodology used was the compilation of information by the Ministry of Economic and Social Inclusion (MIES) Zone 8, on the credits made in the last nine years, with an analysis of the data to conclude with an explanation of the trend of The Human Development credits, where the results highlight the area, district, type, subsidy, sex, age and activity of the families that received the Human Development Credit. The results from research we have that the total of people surveyed the $94 \%$ are women and $6 \%$ are men, the age of $75 \%$ (majority) of mothers are between 18 and 40 years, the greatest activity is concentrated in $58 \%$ of clothing sales, $25 \%$ in breeding Of poultry, cattle breeding, marine aquaculture, marine fishing, and finally the $13 \%$ in food sales.
\end{abstract}

Key words: association human development credit (CDHA); popular and solidarity economy; social inclusion; entrepreneurship 


\section{Introducción}

\section{Evolución Social en América Latina}

Para el buen vivir de los pueblos es importante el desarrollo social, que depende de los modelos que tiene cada uno de los Estados, todos estos modelos pueden aplicarse en un ambiente democrático con un sistema capitalista, pero estos no son eficientes, porque llevan a algunos países a excluir de la vida política a las personas vulnerables, por el hecho de no ser económicamente representativas.

\section{Evolución Reciente de la Pobreza en América Latina}

La tasa de pobreza en América Latina se situó en el 28,2\% en 2014 y la tasa de indigencia alcanzó al 11,8\% del total de la población. El número de personas pobres creció en 2014, alcanzando a 168 millones, de las cuales 70 millones se encontraban en situación de indigencia (CEPAL, 2015).

Este crecimiento tuvo lugar básicamente entre las personas pobres no indigentes, cuyo número pasó de 96 millones en 2013 a 98 millones en 2014 (véase el gráfico 1).

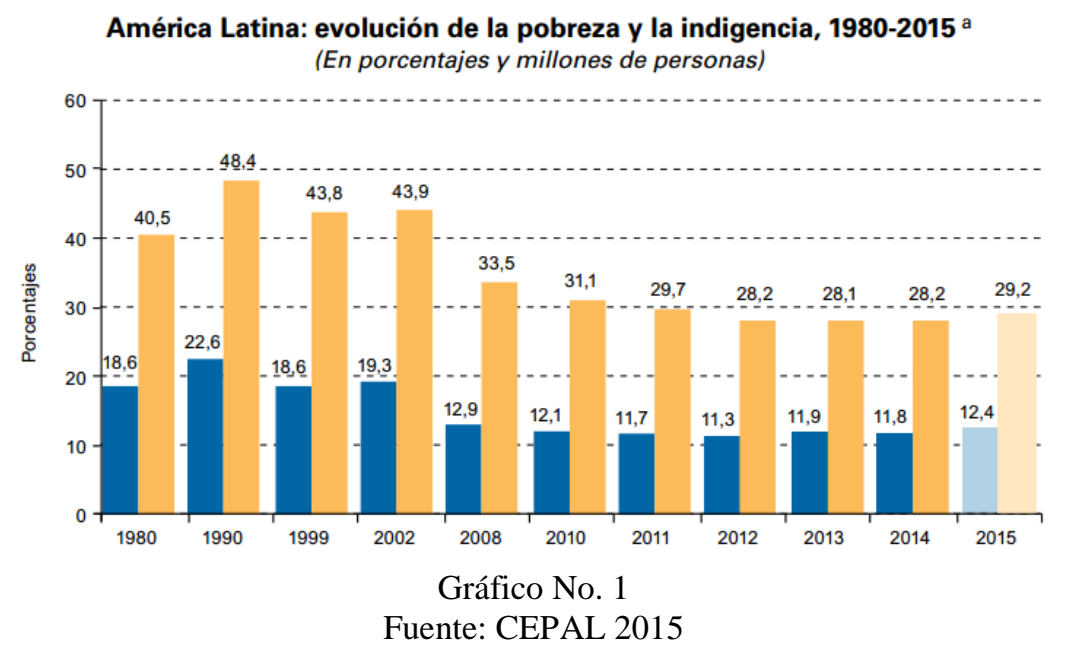

Los cambios de la tasa de pobreza regional provienen de las variaciones observadas en los países, así como de las proyecciones que se efectúan sobre los países en los que no hay datos disponibles de un año determinado. (CEPAL, 2015)

Entre 2013 y 2014, el número de pobres de la región se incrementó en alrededor de 2 millones de personas. Esta variación fue el resultado, por una parte, de un aumento de aproximadamente 7 millones de personas pobres registrado o proyectado principalmente en Guatemala, México y Venezuela (República Bolivariana de), y, por otra, de una disminución de 5 millones observada sobre todo en el Brasil, Colombia y el Ecuador. (CEPAL, 2015) 
Según las proyecciones, en 2015 ambos indicadores varían al alza. La tasa de pobreza se situaría en el $29,2 \%$ y la tasa de pobreza extrema en el $12,4 \%$, lo que representaría aumentos de 1,0 y 0,6 puntos porcentuales, respectivamente. (CEPAL, 2015)

De confirmarse estas proyecciones, 175 millones de personas se encontrarían en situación de pobreza por ingresos en 2015, 75 millones de las cuales estarían en situación de indigencia. (CEPAL, 2015)

A lo largo de los años, la economía mundial experimenta cíclicamente profundas crisis en el sistema capitalista. Si bien se ha centrado principalmente en Europa, en América Latina se ha sobrellevado con resultados favorables en el desarrollo social, evidenciándose una lucha paulatina orientada en la reducción de la pobreza.

La pobreza es una situación estructural que impide a las personas la satisfacción de sus necesidades básicas, excluyéndolas del umbral mínimo para la expansión de sus potencialidades humanas. La pobreza constituye, como tal, la carencia y negación de los prerrequisitos para el desarrollo humano. (Carlos Larrea M., 2004)

A los procesos de independencia y constitución de estados nacionales en América Latina siguieron procesos de desarrollo impulsados y controlados por las oligarquías nacionales. Estos se desenvolvieron en el marco de democracias liberales y tuvieron por objetivo el desarrollo capitalista y la integración con los mercados externos. Pero estas democracias excluyeron de la vida política a las masas populares, privándolas de canales de participación social o de presión política. (Max-Neef, Manfred \& Antonio Elizalde \& Martín Hopenhayn, 2010)

América Latina sigue siendo la región más desigual del mundo, no sólo en relación a los ingresos monetarios sino también en las dimensiones de género, etnia, clase y ubicación territorial. Aunque de manera global los índices de la región han mejorado considerablemente. (Magdalena Sepúlveda, 2014)

Se toma como ejemplo de lo mencionado en el párrafo anterior, respecto a las mejoras de la región, a Colombia que cuenta con varias iniciativas públicas que se dirigen a financiar el nacimiento de nuevas empresas, aunque con mayor focalización en la microempresa que en las empresas con alto potencial de crecimiento. La principal iniciativa es el Fondo Emprender, un instrumento para fomentar y financiar la creación de empresas lideradas por grupos especiales de la población (aprendices o asociaciones entre aprendices, estudiantes universitarios y profesionales, en las que la mayoría sean aprendices) (Echecopar G., \& Angelelli P., \& Galleguillos G.,\& Schorr M., 2006)

Es importante señalar que el microcrédito supone un cambio por parte de la ayuda al desarrollo: en vez de proporcionar fondos no reembolsables, busca que las personas puedan por sí mismas construir una salida a su situación de pobreza. Sirva como ejemplo el que la Agencia Española de Cooperación Internacional (AECI) puso en marcha en 1998 un fondo para concesión de microcréditos, al cual ha destinado hasta finales de 2003 más de 220 millones de Euros. (Gutiérrez G., 2005) 
Por tanto, el microcrédito así definido, no se concentra únicamente en la atención de sujetos pobres (aunque este haya sido el objetivo de los microcréditos en sus inicios). La actuación del sector micro financiero, sobre todo en Latinoamérica, ha evolucionado de tal forma que en la actualidad se orienta a la atención de empresas con acceso insuficiente a servicios financieros y a sectores sin acceso a servicios bancarios, incluidos individuos pobres, y aquellos ubicados por encima de la línea de pobreza, considerando así una base más amplia de clientes. (BERGER, M., 2007)

\section{Evolución Social en Ecuador}

En el Ecuador existen diferentes iniciativas públicas para orientar el desarrollo social entre los cuales se encuentran el Bono de Desarrollo Humano entregado a familia en condiciones de vulnerabilidad y extrema pobreza, otra de las acciones que hizo el Gobierno como política pública es la Promoción y Movilidad Social instrumentado a través del Crédito de Desarrollo Humano tanto el individual como el asociativo y destinado a beneficiarios del bono de desarrollo humano, adultos mayores y personas con discapacidad. (MIES, 2015)

En el año 2007 en el Ecuador se abren espacios al desarrollo y acción social, tomando un nuevo enfoque basado en el ser humano y a la naturaleza como eje central de la economía. Que permita la subsistencia de las futuras generaciones y fomente un entorno de armonía y el principio de solidaridad, igualdad y equidad para todos.

En Ecuador el desarrollo social se refleja en la economía popular y solidaria que reconoce y da apertura a los grupos sociales vulnerables y de bajos recursos económicos que estaban excluidos del sistema financiero tradicional, para con ello dar lugar a que formen parte de la nueva estructura financiera popular y solidaria. Para la nueva Constitución, el Sumak Kawsay implica mejorar la calidad de vida de la población, desarrollar sus capacidades y potencialidades; contar con un sistema económico que promueva la igualdad a través de la re-distribución social y territorial de los beneficios del desarrollo. (Constitución Politica del Ecuador, 2008, pág. art. 276)

En la constitución del 2008, dando respuesta a los requerimientos de los sectores populares, se creó un nuevo sector económico - financiero: el popular y solidario, por lo cual el actual sistema financiero ecuatoriano está formado por tres sectores: público, privado y de la economía popular y solidario, que intermedian recursos del público. (Constitución Politica del Ecuador, 2008, pág. art. 309)

Entre los objetivos del Plan Nacional para el Buen Vivir, que hacen mención al desarrollo local y al emprendimiento a través de la economía popular y solidaria, lo hayamos: el auspiciar la igualdad, la cohesión, la inclusión, y la equidad social y territorial, en la diversidad, y; consolidar el sistema económico, social y solidario, de forma sostenible. (Plan Nacional para el Buen Vivir, 2013, pág. Objetivo 2)

Se entiende por economía popular y Solidaria a la forma económica, donde sus integrantes, individual o colectivamente, organizan y desarrollan procesos de producción, intercambio, comercialización, financiamiento y consumo de bienes y servicios, para satisfacer 
necesidades y generar ingresos, basadas en relaciones de solidaridad, cooperación y reciprocidad, privilegiando al trabajo y al ser humano como sujeto y fin de su actividad, orientada al buen vivir, en armonía con la naturaleza, por sobre la apropiación, el lucro y la acumulación de capital. (Ley Orgánica de la Economía Popular y Solidaria, 2011, pág. art. 1)

\section{El Crédito de Desarrollo Humano en el Ecuador}

La crisis financiera del año 1999 afectó a familias del sector rural en situación de extrema pobreza. Por ello, el Estado creó un bono que mitigaría el impacto económico sobre todo en los grupos sociales más desprotegidos. Desde el 2007 el Instituto Nacional de Economía Popular y Solidaria realizó un nuevo programa de Gobierno con el cual los beneficiarios del Bono de Desarrollo Humano (BDH) reciben el Crédito de Desarrollo Humano (CDH) con la finalidad de desarrollar, acrecentar y potenciar las actividades de producción, y microemprendimiento de los usuarios del Bono.

El crédito de desarrollo humano - $\mathrm{CDH}$ es un servicio financiero que ofrece el gobierno nacional, a través del MIES, a las personas usuarias de la transferencia monetaria conocida como Bono de Desarrollo Humano (BDH), consiste en el pago anticipado de uno o hasta dos años del $\mathrm{BDH}$, a solicitud del usuario, sin otro requisito que hacer la solicitud para obtener un turno para la recepción del crédito, este servicio está vigente desde 2007, en estos años se han entregado más de un 1.300.000 créditos por un monto superior a los 750 millones de USD. (Ministerio de Inclusión Económica y Social, 2013)

El objetivo de este crédito ha sido impulsar emprendimientos productivos y asociativos por parte de sus beneficiarios, de manera que los ingresos consigan superar el valor recibido por el bono (Coba Molina \& Díaz Cordova, 2014)

Un balance de nuestras debilidades y de nuestras fortalezas, se han transformado a lo largo del tiempo en oportunidades de vida mejor para cerca de un millón de personas, en organizaciones que siguen funcionando, en finanzas populares que promueven la producción y derrotan la usura, en comercio justo, en aportes culturales y educativos, en salud, agua y vivienda para decenas de miles de familias. Hemos invertido y gastado bien lo recibido. (Alberto Acosta \& Freddy Alvarez \& Alicia Arias \& Franklin canelos, 2011)

\section{Instituto Nacional de Economía Popular y Solidaria (IEPS)}

IEPS debe proponer e implementar políticas y normas para el desarrollo de la EPS, que contribuyan a generar condiciones para el buen vivir, deberá considerar las potencialidades de los territorios, los enfoques de género, interculturalidad, intergeneracional, ambiental, étnicos y culturales. Así mismo, el literal e) señala que debe organizar y gestionar programas y proyectos de ejecución directa dirigidos al desarrollo de la economía popular y solidaria, y verificar el cumplimiento de los objetivos previstos. (Estatuto Orgánico de gestión del IEPS, 2009, pág. ART. 3)

\section{Unidad de Inclusión Económica (UIE)}


Esta Unidad implementa políticas, planes, programas y proyectos que fomenten emprendimiento y empleo en pro de las familias que reciben el Bono de Desarrollo Humano y promover la articulación de oportunidades productivas, comercio y servicios existentes en el territorio, para la inclusión económica de la población en pobreza y extrema pobreza.

\section{CDH Individual}

El usuario recibe un valor de \$600,00 luego de haber participado en la sesión “Aprendiendo a Emprender" con los técnicos de la Unidad de inclusión Económica del MIES quienes le guían para que pueda iniciar su negocio, puede acceder al CDH Individual solicitando directamente al Call Center o presentando en las Unidades de inclusión Económica del MIES.

\section{CDH Asociativo}

El MIES a través de las Unidades de Inclusión Económica (UIE) y el Instituto de Economía Popular y Solidaria (IEPS), impulsan los créditos asociativos con la finalidad que estos recursos sean invertidos para desarrollar actividades productivas nuevas o en marcha en conjunto con otras personas. Pueden recibir hasta $\$ 1.200,00$ para invertir en materia prima, insumos, mercaderías, equipos y herramientas o en la comercialización. Para acceder al CDH deberán asistir a los talleres que ofrecen el MIES junto con la Universidad de Guayaquil.

El crédito de desarrollo humano $\mathrm{CDH}$ es una ayuda que el gobierno Central ofrece, a través del MIES, a personas que se encuentran en la pobreza extrema y beneficiarias del Bono de Desarrollo Humano (BDH), es el pago por anticipado de hasta dos años del BDH, equivalentes a $\$ 1.200$, a solicitud del usuario, y para crear o mantener un negocio donde puedan generar ingresos que cubran el BDH recibido.

\section{Crédito de Desarrollo Humano en la Zona 8}

La Zona de Planificación 8 está al suroccidente de Ecuador, en la región Costa, provincia del Guayas. Comprende 4 864,55 km2 de superficie y tiene 2654274 habitantes, que representan el 18\% de la población del país; de ésta, el 96\% es urbana (INEC, 2010a). Está conformada por tres cantones: Guayaquil, Samborondón y Durán; seis parroquias rurales, distribuidas de la siguiente manera: Juan Gómez Rendón, El Morro, Posorja, Tenguel, Puná (cantón Guayaquil) y Tarifa (cantón Samborondón). En la Zona 8 se han conformado 12 distritos y 67 circuitos administrativos. (BUEN VIVIR PLAN NACIONAL, 2017)

La Coordinación zonal 8 (Guayas, los cantones de Guayaquil, Durán y Samborondón) del Ministerio de Inclusión Económica y Social (MIES), el Gobierno zonal está ubicado (Av. Francisco de Orellana) al edificio Makro, ubicado en la Av. Carlos Luis Plaza Dañín y calle Francisco Boloña.

En este edificio, el MIES da los servicios en lo que respecta a los programas de Bono de Desarrollo Humano, Desarrollo Infantil, Protección Especial, Atención Integral a Adultos Mayores y Personas con Discapacidad, Juventudes, Instituto de Economía Popular y Solidaria y asesoría jurídica para el registro de cooperativas y asociaciones. (MIES, 2017) 
En los últimos 15 años, la periferia de la ciudad de Guayaquil ha crecido, su rápida expansión no ha demostrado un buen desarrollo, por la falta de planes y estrategias por parte de los organismos locales, lo que ocasiona que sus habitantes vivan en pobreza y extrema pobreza.

\section{Metodología}

Para realizar el presente diagnóstico se tomaron datos del Ministerio de Inclusión Económica y Social de la zona 8, donde se mostrará con gráficos estadísticos el comportamiento de los beneficiarios del Crédito de Desarrollo Humano entre los años 2007 al 2016, el primer lugar será un análisis bibliográfico de los últimos nueve años del 2007 al 2015, cuyas variables de los beneficiarios del CDH serán, por subsidio, por distrito y por tipo de $\mathrm{CDH}$. El segundo lugar será análisis del año 2016 de los primeros ocho meses, tomando en consideración las variables: por sexo, por distritos, tipos de beneficiarios, por edades, por zona, por actividad.

Con alumnos de la Universidad de Guayaquil se constató el entorno socioeconómico de los beneficiarios del $\mathrm{CDH}$. Ha de indicarse que las familias visitadas estaban ubicadas en zonas urbanas marginales de la ciudad, dándose ocasiones en las que para encuestar y verificar la existencia de las actividades de emprendimiento se toma uno o dos horas para llegar al lugar de destino, donde el transporte no llega. Con lo antes mencionado se pretende subrayar el entorno de pobreza y exclusión.

\section{Resultados}

Por tal razón el Gobierno se ha enfocado en dar el Crédito de Desarrollo Humano (CDH) a los habitantes asentados en áreas urbanas marginales de la zona 8. En el grafica No. 2 se observa que desde el 2007 este crédito se ha ido incrementando progresivamente en los siguientes años, hasta tener un repunte en el año 2010 con un total de beneficiarias de 64.687, las razones de este incremento se debe al censo Social realizado por Ministerio Coordinador de Desarrollo Social. En el año 2008, se depuraron los listados de los beneficiarios del Bono de Desarrollo Humano, en el cual hubo un incremento de Madres, Adulto mayores y Personas con discapacidad, los mismo que accedían al crédito de manera fácil, ingresando a la página web del Ministerio de Inclusión Económica y Social y sin que se lleve a cabo el control y seguimiento de los emprendimientos requeridos.

En el año 2010 se generó un incremento acelerado de la demanda de créditos individuales, debido a que el plazo se extendió hasta 24 meses. Hasta ese momento el valor del CDH era de USD 840, por esta razón solicitaban los créditos de forma masiva, otra razón fue que se rumoraba que para el siguiente año el crédito se lo limitaría, dándose sólo a beneficiarios que lleven a cabo sus emprendimientos para lo cual asistirían a talleres de capacitación.

Para el año 2012 hubo un incremento debido a la participación del Sector Popular y Solidario que incentivaban a los usuarios a obtener un $\mathrm{CDH}$. 


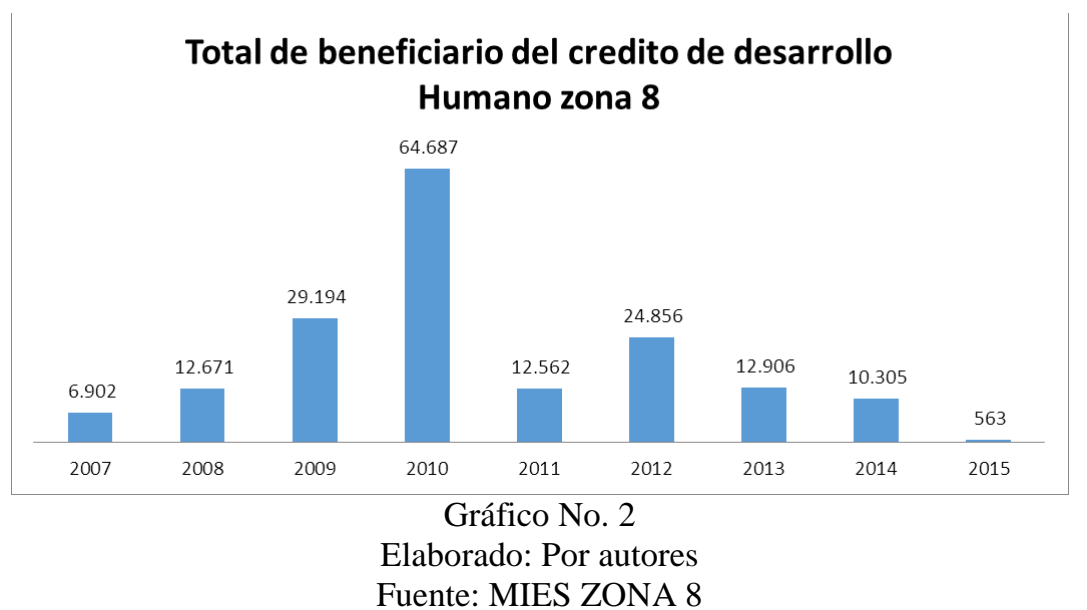

Si a estos mismos beneficiarios lo relacionamos con el tipo de subsidio (BDH - Adulto mayores - Personas con discapacidad), durante el mismo periodo podemos establecer que el $91 \%$ de los beneficiarios de CDH fueron entregados a familias que recibe el Bono de Desarrollo Humano y que se encuentra en condiciones de extrema pobreza en su mayoría son madres solteras, $7 \%$ para adultos mayores y el $2 \%$ a personas con discapacidad, como lo indica en el Gráfico No. 3.

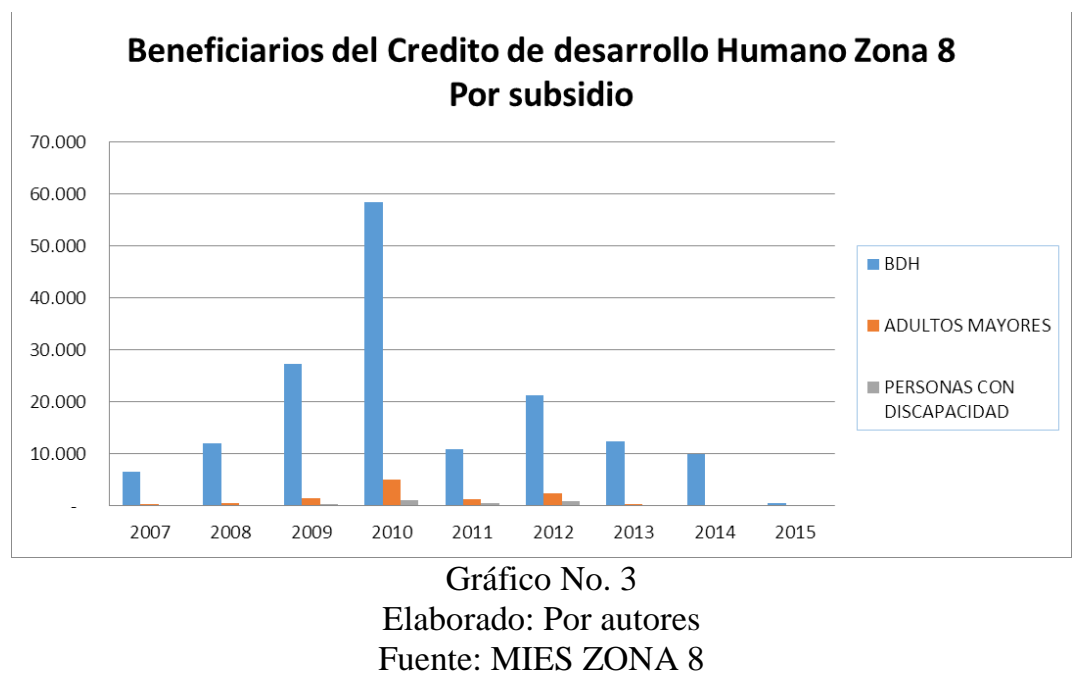

Los sectores de la zona 8 con mayor vulnerabilidad y donde el Gobierno Nacional se ha enfocado para entregar el Crédito de Desarrollo Humano $(\mathrm{CDH})$ son: sector Norte (Monte Sinaí, Voluntad de Dios, Sergio Total, Valerio Estacio, Nueva Prosperina, Socio Vivienda, Flor de Bastión, entre otros, pertenecientes a la Parroquia Tarqui), sector Sur (Guasmo, Isla trinitaria, suburbio, Independencia II entre otros, de la parroquia Ximena). Sector Duran (Recinto La Unión, Recreo, El Mirador, Isla Santay, Gregorio II, entre otros)

En el Grafico No. 4 podemos observar que el 59\% de los beneficiarios del CDH corresponde al sector Norte; principalmente en las Parroquias de Tarqui y Pascuales, el 23\% al sector Norte y el $18 \%$ al sector de Duran y Samborondon. 
En términos monetarios para el año 2016, el estado tuvo un mayor desembolso en el sector Norte de Guayaquil con un valor de \$ $2670.744,56$ USD, con relación a los demás sectores, debido a la expansión de los asentamientos irregulares del sector Norte.
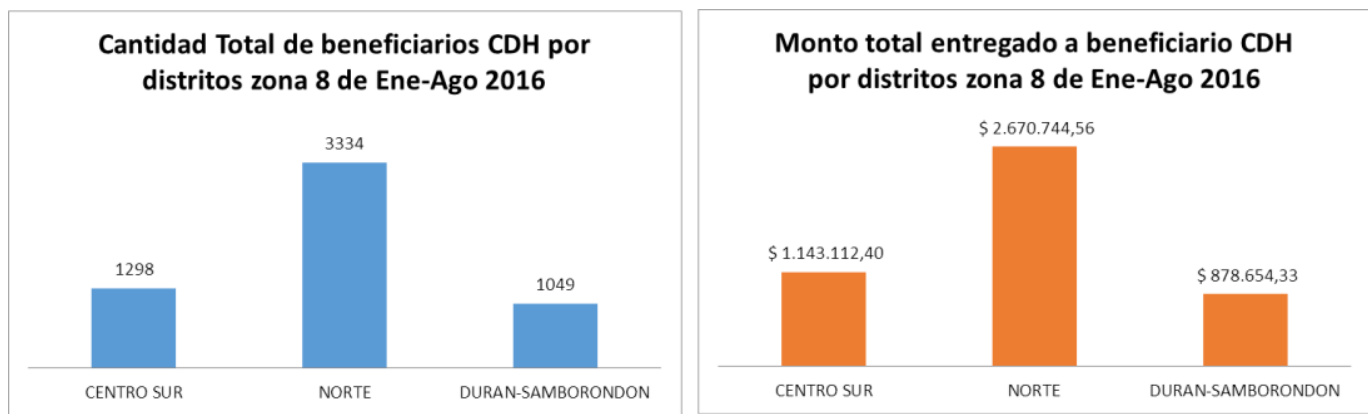

Gráfico No. 4

Elaborado: Por autores

Fuente: MIES ZONA 8

Para el Ecuador, el nuevo modelo económico da gran prioridad a los procesos productivos del sector popular y solidario, determina el potencial del micro-finanzas en el empoderamiento de las personas emprendedoras de este sector.

En el grafico No. 5. El mayor desembolso del crédito de desarrollo humano (CDH) se lo hace de manera Asociativo UIE, por USD \$2 $935.218,40$ que representa el $63 \%$ del total y sigue el crédito individual por $\$ 1^{\prime} 694.712,40$ con un $36 \%$, y por último crédito asociativo IEP con el $1 \%$ por el valor $\$ 62.580,00$, lo que da a entender que los beneficiarios pone mucho énfasis en la asociatividad en el inicio de su negocio.
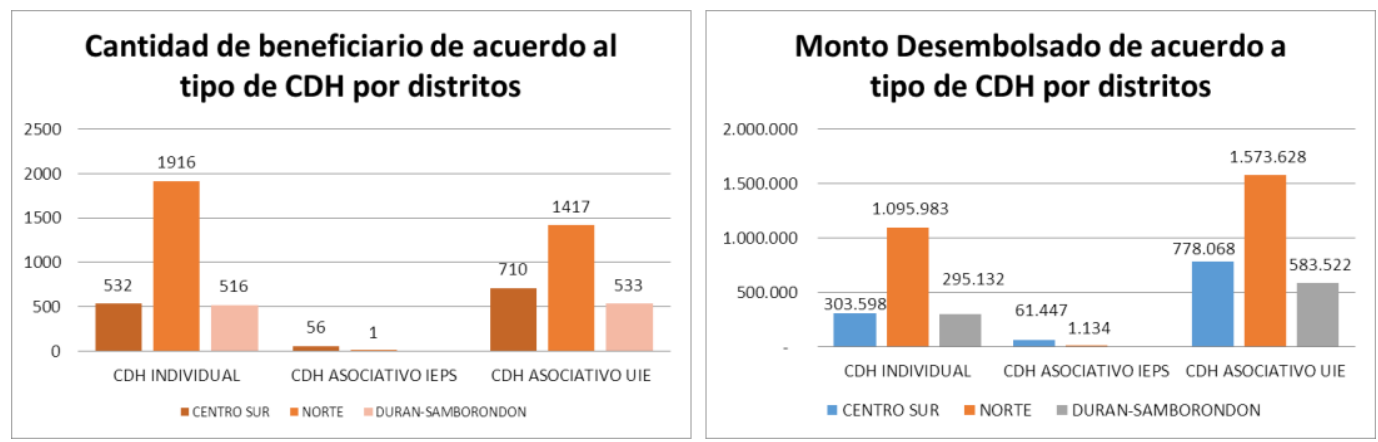

Gráfico No. 5

Elaborado: Por autores

Fuente: MIES ZONA 8

En el presente año, el MIES tiene previsto trabajar en la zona 8 con 6.261 familias beneficiarias del bono de desarrollo humano, orientadas a la inclusión económica y movilidad social ascendente, A través de la implementación de políticas, planes, programas y proyectos que fomenten emprendimiento y empleo en las familias que reciben el BDH y promover la actividades productivas, comercio y servicio del lugar de la población en pobreza y extrema pobreza. 
Una vez cumplidas estas actividades, se procederá con la generación de emprendimientos y mecanismos de financiamiento, que promuevan la articulación de oportunidades productivas, comercio y servicios existentes en el territorio, para la inclusión económica de la población en pobreza y extrema pobreza.

Según dato del MIES zona 8 existe un desembolso total de Crédito de Desarrollo Humano en el año 2016 de USD \$ 5’058.189,99 entregados a 6.261 beneficiarios cuyo comportamiento mensual de dichos desembolsos es el siguiente: Enero \$114.449,12 (2\%), Febrero \$ 924.894,29 (18\%), Marzo \$ 813.288,46 (16\%), Abril \$ 823.237,43 (16\%), Mayo \$ 74.268,76 (1\%), Junio \$ 708.032,00 (14\%), Julio \$ 1.124.912,63 (22\%), Agosto \$ 475.107,30 (9\%). Cabe indicar que los meses de Enero y Mayo fueron atípicos debido a que en Enero se demoraron las asignaciones por inicio del ejercicio fiscal y en mayo bajaron los créditos debido al terremoto del 16 de abril del 2016.

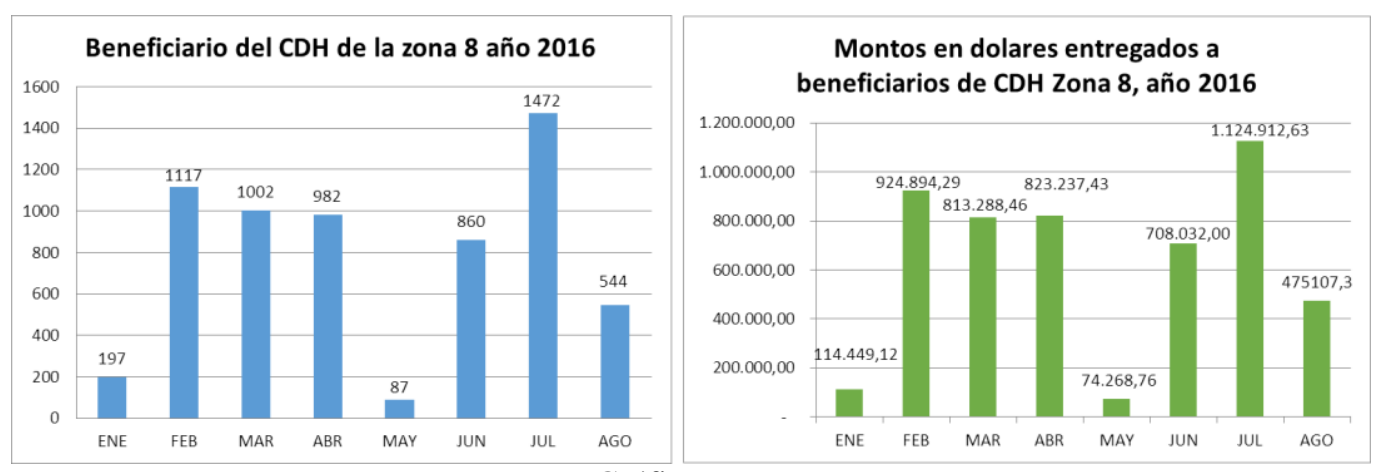

Gráfico No. 6

Elaborado: Por autores

Fuente: MIES ZONA 8

En el grafico No. 7, de los 6.261 beneficiarios del CDH en el año 2016, si lo clasificamos por sexo (hombre y mujer) observamos que el $94 \%$ de los créditos entregados corresponde a mujeres y el $6 \%$ corresponde a hombre. Podemos decir que los emprendimientos son realizados más por mujeres debido a la necesidad que tienen de obtener ingresos para la manutención de sus hijos, en mucho de los casos son madres solteras abandonadas por su pareja.

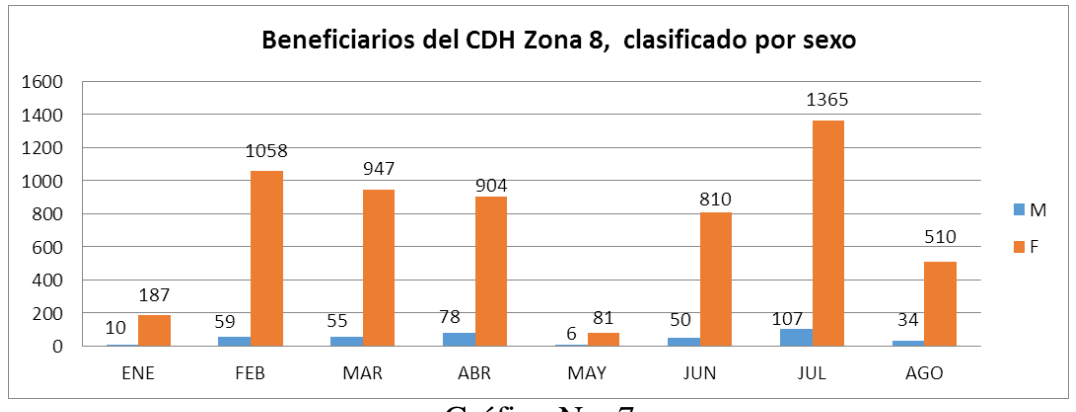

Gráfico No. 7

Elaborado: Por autores

Fuente: MIES ZONA 8 
En el grafico No. 8 de las 6.261 beneficiarias del CDH 96,44\% corresponde a Madres beneficiarias del BDH, EL 2,22\% adulto mayores y el 1,34\% personas con discapacidad. Para ser beneficiario del Crédito de desarrollo Humano deben ser madres en condiciones de extrema pobreza beneficiaria de BDH, adulto mayor que reciba una pensión, persona con discapacidad que reciba una pensión y que tengan ingresos muy bajos y así cumplir con los requisitos del crédito.

Las madres solteras, separadas o divorciadas, no tienen una relación de pareja convencional, lo que las hace enfrentar los gastos por el sostenimiento de su hogar solas y en algunos casos se convierte en un limitante individual y social que las impide iniciar una nueva relación de pareja. Las mujeres viudas no viven el rechazo y el estigma que viven las madres solteras, separadas o divorciadas, aunque al igual que éstas, afrontan solas los gastos de la casa y a pesar de que viven sin sus esposos, muchas de ellas logran rehacer sus vidas con una nueva pareja. (Lobelia J. Teresa de J. Jorge E., 2016)

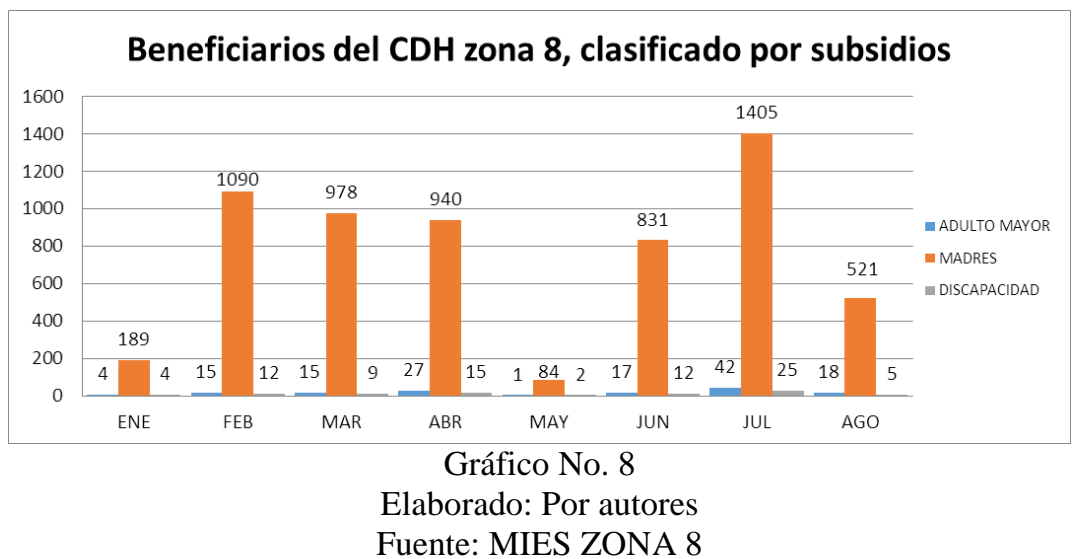

En el gráfico No. 9, De acuerdo a la edad de los beneficiarios del CDH el 31,23\% reflejo que están entre los 18 a 30 años de edad, el 44,16\% se encuentran entre los 31 y 40 años, el $15,27 \%$ entre 41 y 50 años, el $5,73 \%$ entre los 51 y 60 años y finalmente el 3,61\% restante pertenece a las personas de 61 añps de edad en adelante. Lo que demuestra que el $75 \%$ de madres son jóvenes y están en el grupo de la población económicamente activa, que por falta de oportunidades y estar a cargo de la manutención de sus hijos no puede conseguir un empleo pleno.

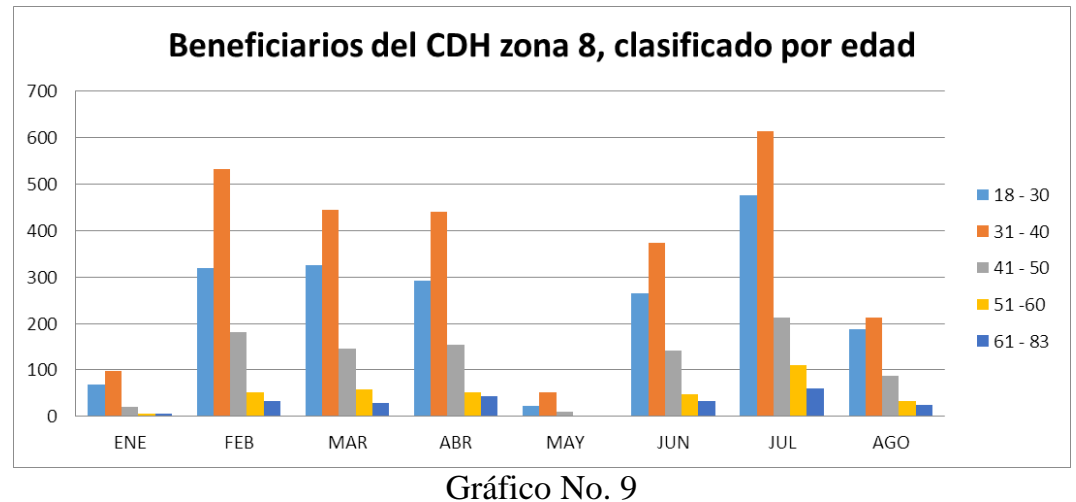


Elaborado: Por autores

Fuente: MIES ZONA 8

En el grafico 10 de lo beneficiario del BDH el 84\% están en la zona urbana de Guayaquil como la parroquia Tarqui, Ximena, Febres cordero, letamendi, Eloy Alfaro Duran, samborondon, Simón bolívar, Alfredo Baquerizo y 15,94\% zona rural como Tenguel, Tarifa, Puna, Juan Gómez Rendón, Mariscal Sucre, San Carlos, Coronel Llerena y otros.

Grafico No. 10

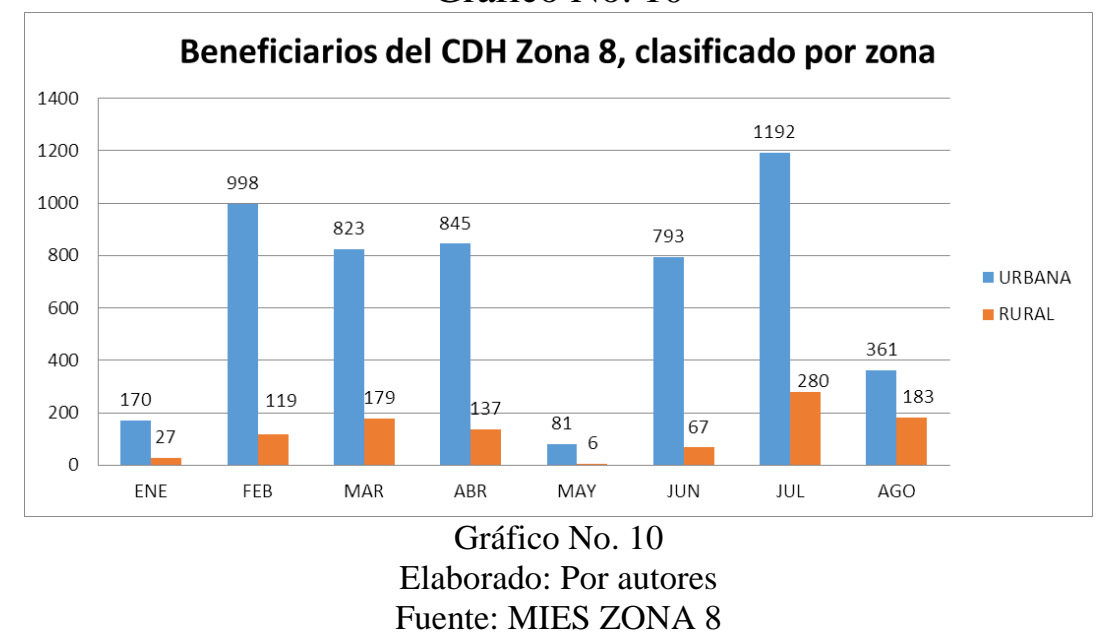

En el gráfico 11 se observa que de las actividades que desarrollan los beneficiarios del $\mathrm{CDH}$ de la zona 8 , el $13 \%$ a actividades de alojamiento y de servicio de comidas (restaurante), el $25 \%$ a agricultura, ganadería, acuicultura y pesca (cría de ave de corral, cría de ganado bovino, acuicultura marina, pesca marina), el 58\% al comercio por mayor y menor, reparación de vehículos automotores y motocicletas (venta de ropa), el $2 \%$ industria manufacturera (elaboración de alimentos, fabricación de prendas de vestir y otros) y el $1 \%$ a otras actividades de servicio (lavado y limpieza, peluquería).

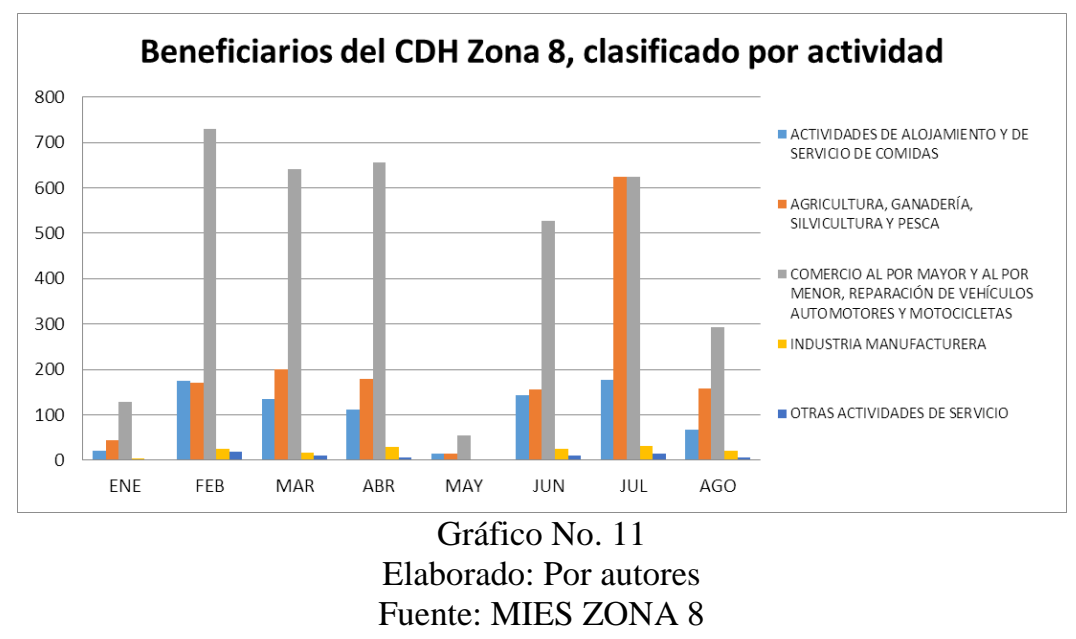

Conclusiones 
Dentro de la evolución social vemos que a lo largo de los años, la economía mundial experimenta una profunda crisis en el sistema capitalista. No solo se ha centrado en Europa, sino también en América Latina donde se ha sobrellevado con resultados favorables en el desarrollo social, evidenciándose una lucha paulatina a favor de la reducción de la pobreza.

Este trabajo es una primera evaluación a través de datos obtenidos por el MIES en la zona 8; la investigación puede ampliarse a varias fases de estudio. El Gobierno se ha enfocado en dar el Crédito de desarrollo Humano $(\mathrm{CDH})$ a los habitantes asentados en área urbana marginal de la zona 8 donde se ve el comportamiento desde 2007 al 2010 que tenía sus altas y bajas de acuerdo a las razones detalladas en el artículo. Durante todo este tiempo se pudo establecer el $91 \%$ de los beneficiarios de $\mathrm{CDH}$ fueron entregados a familias que recibe el Bono de Desarrollo Humano y que se encuentra en condiciones de extrema pobreza en su mayoría son madres solteras, 7\% para adultos mayores y el $2 \%$ a personas con discapacidad. También podemos observar que el $59 \%$ de los beneficiarios del CDH corresponde al sector Norte de la ciudad de Guayaquil donde tuvo un desembolso para el crédito de \$ $2670.744,56$ una de las razones es la expansión de los asentamientos irregulares.

Según datos proporcionados por el MIES Zona 8 existe un desembolso total de Crédito de Desarrollo Humano en el año 2016 de USD \$ 5’058.189,99 entregados a 6.261 beneficiarios, si lo clasificamos por sexo (hombre y mujer) observamos que el $94 \%$ de los créditos entregados corresponde a mujeres y el $6 \%$ corresponde a hombre. Podemos decir que los emprendimientos son realizados en su mayoría por mujeres debido a la necesidad que tienen de generar ingresos para mantener a sus hijos. Si lo clasificamos por subsidio tenemos que $96,44 \%$ corresponde a Madres beneficiarias del BDH, El 2,22\% adulto mayores y el 1,34\% personas con discapacidad.

La edad de los beneficiarios del CDH son: El 75\% de las madres son jóvenes entre las edades de 18 y 40 años y se las consideran población económicamente activa, por falta de oportunidades y tener que cuidar a sus hijos no puede conseguir un pleno empleo. También podemos decir que el $84 \%$ se encuentran en la Zona Urbana de Guayaquil como la parroquia Tarqui, Ximena, Febres Cordero, Letamendi, Eloy Alfaro Duran, Samborondón, Simón bolívar, Alfredo Baquerizo y 15,94\% zona rural como Tenguel, Tarifa, Puna, Juan Gómez Rendón, Mariscal Sucre, San Carlos, Coronel Llerena y otros.

Las actividades que desarrollan los beneficiarios del CDH de la zona 8 son: el $58 \%$ al comercio por mayor y menor, (venta de ropa), el $25 \%$ a agricultura, ganadería, acuicultura y pesca (cría de ave de corral, cría de ganado bovino, acuicultura marina, pesca marina), el $13 \%$ a actividades de servicio de comidas (restaurante), el 2\% industria manufacturera (elaboración de alimentos, fabricación de prendas de vestir y otros) y el $1 \%$ a otras actividades de servicio (lavado y limpieza, peluquería). Siendo como referente los negocios de comercio, agricultura y ganado, y comida de mayor acogida; dado que los emprendedores tienen cercanía, considerable conocimiento y mejor desenvolvimiento en ello; motivo del cual a futura investigación se puede profundizar en los tipos de negocio no tradicionales y su falta de incentivos por parte de las instituciones del sector productivo para que el emprendedor ecuatoriano realice el esfuerzo por incursionar en nuevas líneas de negocio. 


\section{Bibliografía}

Alberto Acosta \& Freddy Alvarez \& Alicia Arias \& Franklin canelos. (2011). Debates Sobre Cooperacion Y Modelos Perspectivas Desde La Sociedad Civil En El Ecuador. Centro De Investigaciones Cilidad Observatorio de la Cooperaci6n al Desarrollo en Ecuador, 22.

BERGER, M. (2007). El boom de las microfinanzas. El modelo latinoamericano visto desde adentro. Banco Interamericano de Desarrollo BID, Washington D.C., 1-42.

Buen Vivir Plan Nacional. (18 de 03 de 2017). Agenda resumen ¿Zona 8: Guayaquil, Durán y Samborondón. Recuperado el 18 de 03 de 2017, de Agenda resumen ¿Zona 8: Guayaquil, Durán y Samborondón: http://www.buenvivir.gob.ec/agenda-zona8

Carlos Larrea M. (2004). desarrollo humano en Ecuador. Dosier, 50.

CEPAL. (2015). Panorama Social De America Latina. Naciones Unidas.

Coba Molina \& Díaz Cordova. (2014). El crédito de Desarrollo Humano Asociativo, en la economía social y solidaria de la provincia de Tungurahua - Ecuador. Analitika, Revista de Analisis Estadístico, 33 - 47.

Constitución Politica del Ecuador. (2008). ART. 276. Quito, Pichincha, Ecuador: Registro Oficial.

Echecopar G., \& Angelelli P., \& Galleguillos G.,\& Schorr M. (2006). Capital semilla para el financiamiento de las nuevas empresas . Avances y lecciones de America latina, 11.

Estatuto Orgánico de gestión del IEPS. (2009). Estatuto Orgánico de gestión del IEPS. Estatuto Orgánico de gestión del IEPS. Quito, Pichincha, Ecuador: Ninguna.

Gutiérrez G. (2005). Antecedentes delmicrocrédito. Lecciones delpasado para lasexperiencias actuales. CIRIEC-España, Revista de Economía Pública, Socialy Cooperativa, 29.

Ley Orgánica de la Economía Popular y Solidaria. (2011). Ley Orgánica de la Economía Popular y Solidaria. Artículo 1 . Quito, Pichincha, Ecuador: Ninguna.

Lobelia J. Teresa De J. Jorge E. (2016). Analisis De Los Microemprendimientos Dirigidos Por Mujeres En Un Sector Popular De La Ciudad De Guayaquil- Ecuador. Obtenido De Analisis De Los Microemprendimientos Dirigidos Por Mujeres En Un Sector Popular De La Ciudad De Guayaquil- Ecuador: http://www.eumed.net/cursecon/ecolat/ec/2016/mujeres.html

Magdalena Sepúlveda. (2014). De la retórica a la práctica: el enfoque de derechos en la protección social en América Latina. Políticas Sociales, 61.

MASLOW. (2004). Principios De La Administracion. España: Planeta.

Max-Neef, Manfred \& Antonio Elizalde \& Martín Hopenhayn. (2010). Desarrollo a escala humana Opciones para el futuro. 13. 
MIES. (2015). Bono De Desarrollo Humano. Obtenido de http://www.inclusion.gob.ec/

MIES. (18 de 03 de 2017). Coordinación Zonal 8 Del Mies En Nueva Dirección. Recuperado el 18 de 03 de 2017, de Coordinación Zonal 8 Del Mies En Nueva Dirección: http://www.inclusion.gob.ec/coordinacion-zonal-8-del-mies-en-nueva-direccion/

MIES. (18 de 03 de 2017). Más de 9 mil emprendedores accedieron al Crédito de Desarrollo Humano en la Zonal 8 en 2016. Recuperado el 18 de 03 de 2017, de Más de 9 mil emprendedores accedieron al Crédito de Desarrollo Humano en la Zonal 8 en 2016: http://www.inclusion.gob.ec/mas-de-9-mil-emprendedores-accedieron-al-credito-dedesarrollo-humano-en-la-zonal-8-en-2016/

Ministerio de Inclusión Económica y Social. (2013). Lineas Innovadoras Para Aceso A Credito De Sectores En Situacion De Extrema Pobreza. Obtenido de http://www.finanzaspopulares.gob.ec

Plan Nacional para el Buen Vivir . (2013). Plan Nacional para el Buen Vivir 2013-2017. Objetivo 2. Quito, Pichincha, Ecuador: SENPLADES. 